\title{
Identification of biomarkers associated with the recurrence of osteosarcoma using ceRNA regulatory network analysis
}

\author{
SHANYONG ZHANG ${ }^{1}$, LEI DING $^{2}, \mathrm{XIN} \mathrm{LI}^{3}$ and HONGWU FAN ${ }^{4}$ \\ Departments of ${ }^{1}$ Spinal Surgery, ${ }^{2}$ Rehabilitation, and ${ }^{3}$ Joint Surgery, \\ The Second Hospital of Jilin University, Changchun, Jilin 130041; ${ }^{4}$ Department of Orthopedics, \\ China Japan Union Hospital of Jilin University, Changchun, Jilin 130021, P.R. China
}

Received March 27, 2018; Accepted December 7, 2018

DOI: $10.3892 /$ ijmm.2019.4108

\begin{abstract}
The aim of the present study was to identify the important mRNAs, micro (mi)RNAs and long non-coding (lnc)RNAs that are associated with osteosarcoma recurrence. The GSE3905 dataset, which contains two sub-datasets (GSE39040 and GSE39055), was downloaded from the Gene Expression Omnibus (GEO). Prognosis-associated RNAs were identified by performing Cox regression univariate analysis and were subsequently used to construct a competing endogenous (ce)RNA regulatory network for Gene Set Enrichment Analysis (GSEA). Kaplan-Meier survival analysis was used to determine the associations between expression levels and survival prognosis. In addition, another independent miRNA profile, GSE79181, was downloaded from GEO for validation. Among the differentially expressed RNAs, 417 RNAs (5 lncRNAs, 19 miRNAs, and 393 mRNAs) were observed to be associated with prognosis. The GSEA for the ceRNA regulatory network revealed that 'Mitogen-activated protein kinase (MAPK) signaling pathway', 'Chemokine signaling pathway' and 'Spliceosome' were markedly associated with osteosarcoma. In addition, three lncRNAs [long intergenic non-protein coding RNA 28 (LINC00028), LINC00323, and small nucleolar RNA host gene 1 (SNHG1)] and two miRNAs (hsa-miR-124 and hsa-miR-7) regulating three mRNAs [Ras-related protein Rap-1b (RAP1B), activating transcription factor 2 (ATF2) and protein phosphatase $\mathrm{Mg}^{2+} / \mathrm{Mn}^{2+}$ dependent 1B (PPM1B)] participated in the MAPK signaling pathway. The Kaplan-Meier survival analysis also demonstrated that samples with lower expression levels of LINC00323 and SNHG1 had better prognosis, and samples with increased expression levels of LINC00028, hsa-miR-124 and hsa-miR-7 had better prognosis. Overexpression of RAP1B, ATF2
\end{abstract}

Correspondence to: Dr Hongwu Fan, Department of Orthopedics, China Japan Union Hospital of Jilin University, 126 Xiantai Street, Changchun, Jilin 130021, P.R. China

E-mail: hongwwwwfff@outlook.com

Key words: osteosarcoma, recurrence, competing endogenous RNA network and PPM1B was positively associated with osteosarcoma recurrence. The roles of hsa-miR-124 and hsa-miR-7 in osteosarcoma recurrence were also validated using GSE79181. Thus, in conclusions, the three lncRNAs (LINC00028, LINC00323 and SNHG1), two miRNAs (hsa-miR-124 and hsa-miR-7) and three mRNAs (RAP1B, ATF2, and PPM1B) were associated with osteosarcoma recurrence.

\section{Introduction}

Osteosarcoma is the most common primary malignant bone tumor and often exhibits locally invasive growth. Osteosarcoma is commonly characterized by an appendicular primary tumor with a high rate of pulmonary metastases (1). In addition, osteosarcoma is a genetically unstable and highly malignant mesenchymal tumor of the bone characterized by structural chromosomal alterations $(2,3)$.

Improved understanding of the molecular mechanisms underlying osteosarcoma would support the development of effective and targeted therapies. Many previous studies have reported molecular mechanisms underlying osteosarcoma that were involved in the progression and metastasis of osteosarcoma (4-8). Inhibiting Wnt/ $\beta$-catenin signaling and targeting c-Met, a Wnt-regulated proto-oncogene, have shown to be useful in the treatment of osteosarcoma $(4,5)$. The Notch pathway has a vital role in regulating tumor angiogenesis and vasculogenesis in osteosarcoma (6). Wilms' tumor gene 1 has been reported to act as a tumor promoter in osteosarcoma by regulating cell cycle and apoptotic machinery (7). Recently, COP9 signalosome subunit 3 (COPS3), another oncogene overexpressed in osteosarcoma, was revealed to be involved in osteosarcoma metastasis by interacting with Beclin1 and Raf-1 through autophagy (8). In addition, some studies have reported the roles of long non-coding RNAs (lncRNAs) in the progression, metastasis, and prognosis of osteosarcoma (9-16). A previous study also demonstrated that lncRNAs may function as competing endogenous RNAs (ceRNAs) to sponge microRNAs (miRNAs), thereby regulating miRNA targets (17).

Hedgehog signaling in mature osteoblasts is responsible for the pathogenesis of osteoblastic osteosarcoma through the overexpression of yes-associated protein 1 and lncRNA H19 (9). The IncRNA small nucleolar RNA host gene 1 
(SNHG12) could upregulate the expression of Notch2 to promote osteosarcoma metastasis by acting as a sponge of miR-195-5p (10). The IncRNA focally amplified lncRNA on chromosome 1 has been shown to be a negative prognostic biomarker and exhibit an important pro-oncogenic effect on osteosarcoma progression by targeting epithelial-mesenchymal transition (11). Decreased expression levels of the lncRNA maternally expressed 3 (MEG3) has been reported to be an independent predictor of shorter overall survival in patients with osteosarcoma, suggesting that MEG3 lncRNA may be a useful biomarker for the prognosis of osteosarcoma (12). In addition, lncRNA MEG3 could promote osteosarcoma cell growth and metastasis in vitro by acting as a sponge of miR-127 (13). In a two-stage and case-control study of 900 osteosarcoma cases and 900 controls among the Chinese population, those with the rs7958904 CC genotype had a significantly lower expression level of the lncRNA HOX transcript antisense RNA than those with other genotypes, as well as a lower risk of osteosarcoma (14). The upregulation of lncRNA taurine upregulated 1 was also revealed to be strongly associated with poor prognosis and was an independent prognostic indicator for overall progression-free survival in osteosarcoma (15). Patients with osteosarcoma and high expression levels of lncRNA HOXA distal transcript antisense RNA had poorer overall survival than those with low expression levels (16). The majority of prognostic miRNAs were located at $14 q 32$ in a study by Kelly et al (18); their gene targets displayed deregulation patterns associated with outcomes based on their developed miRNA models, with independent predictive values for recurrence and overall survival from formalin-fixed, paraffin-embedded human osteosarcoma diagnostic biopsy specimens. However, the roles of the lncRNAs were not investigated. In the present study, the GSE3905 dataset was downloaded to identify prognosis-associated RNAs for ceRNA regulatory network construction. In addition, the important pathways were determined using Gene Set Enrichment Analysis (GSEA). Kaplan-Meier survival analysis was conducted to determine the associations between the expression levels and survival prognosis. Furthermore, another independent miRNA profile, GSE79181, was downloaded from Gene Expression Omnibus (GEO) for validation (Fig. 1).

\section{Materials and methods}

Microarray data and preprocessing. The expression profile of GSE39058 (18), containing two subdatasets (GSE39040 and GSE39055), was downloaded from GEO (www.ncbi.nlm.nih. gov/geo/). The small non-coding RNA (miRNA) profile of GSE39040, containing 65 human osteosarcoma biopsy specimens, was generated using the platform GPL15762 (Illumina Human v2 MicroRNA Expression BeadChip; Illumina, Inc., San Diego, CA, USA) and the expression profile of GSE39055 containing 37 human osteosarcoma biopsy specimens was based on the platform of GPL14951 (Illumina HumanHT-12 WG-DASL V4.0 R2 Expression BeadChip); Illumina, Inc.). The paired miRNA and mRNA profiles of 37 biopsy specimens were used for further analysis. The probe names in the gene expression profiles were converted into gene names. In addition, the mean value of a gene symbol matched with multiple probes was calculated using the function in
R software (version 3.4.1) (19). Subsequently, the microarray data were normalized using the preprocessCore package in $\mathrm{R}$ software (version 3.4.1) (19). The Linear Models for Microarray Analysis (Limma) package (version 3.32.5) in R software (20) was used for $\log 2$ conversion and the sequencing data were normalized using the quantile method (21).

Differential expression analysis and hierarchical clustering. The mRNAs and lncRNAs in the GSE39055 dataset were annotated using the Transcript ID, RefSeq ID and location, supported by the GPL14951 (Illumina HumanHT-12 WG-DASL V4.0; Illumina, Inc.) platform and HUGO Gene Nomenclature Committee (www.genenames.org), which has 3,859 lncRNAs and 19,180 protein-coding genes (22). The 37 biopsy specimens of the paired miRNA and mRNA profile were divided into recurrent $(n=18)$ and non-recurrent $(n=19)$ osteosarcoma samples based on the clinical information. The Limma package (20) was used to perform differential expression analysis between recurrent and non-recurrent osteosarcoma samples. The P-values were adjusted for false discovery rate (FDR) using the Benjamini-Hochberg method (23). The thresholds for screening differentially expressed mRNAs, miRNAs and IncRNA were set as FDR $<0.05$ and $\mid \log _{2}$ fold-change $(F C) \mid>0.585$. In addition, the expression values of screened differentially expressed RNAs were hierarchically clustered using the pheatmap package (version 1.0.8) (24) in $\mathrm{R}$ based on the encyclopedia of distances (25) to observe the differences in expression levels spontaneously.

Screening prognosis-associated RNAs. The expression levels of differentially expressed RNAs and the prognostic information of each sample were collected. The survival package (26) in $\mathrm{R}$ was used to identify the prognosis-associated mRNAs, miRNAs and lncRNAs by Cox regression univariate analysis, and $\mathrm{P}<0.05$ was used as the cut-off criterion. Subsequently, the Database for Annotation, Visualization and Integrated Discovery (version 6.8) (27) was used to perform Gene Ontology (GO) functional [biology process (BP), molecular function (MF) and cellular component (CC)] and Kyoto Encyclopedia of Genes and Genomes (KEGG) pathway enrichment analysis for prognosis-associated mRNAs, with a threshold of $\mathrm{P}<0.05$.

ceRNA regulatory network construction and analysis. The identified prognosis-associated lncRNAs and miRNAs were used to select IncRNA-miRNA regulatory relationships from the associations in miRcode (version 11; www.mircode. org/) and starBase (version 2.0; starbase.sysu.edu.cn/index. php) (28). In addition, the miRNA-mRNA regulatory associations were selected based on the regulatory information in TargetScan (release 7.1; www.targetscan.org/vert_71/) (29), miRTarBase (release 6.0; mirtarbase.mbc.nctu.edu.tw) (30), miRanda (version 2.0; www.microrna.org/microrna/home. do) (31) and miRBase (release 21; www.mirbase.org/) (32). The ceRNA regulatory network was constructed by integrating the lncRNA-miRNA and miRNA-mRNA regulatory relationships via Cytoscape (version 3.4; www.cytoscape.org/) (33).

The genes in the constructed ceRNA regulatory network were used for GSEA (2017 update; software.broadinstitute.org/gsea/index.jsp) to identify the important KEGG 


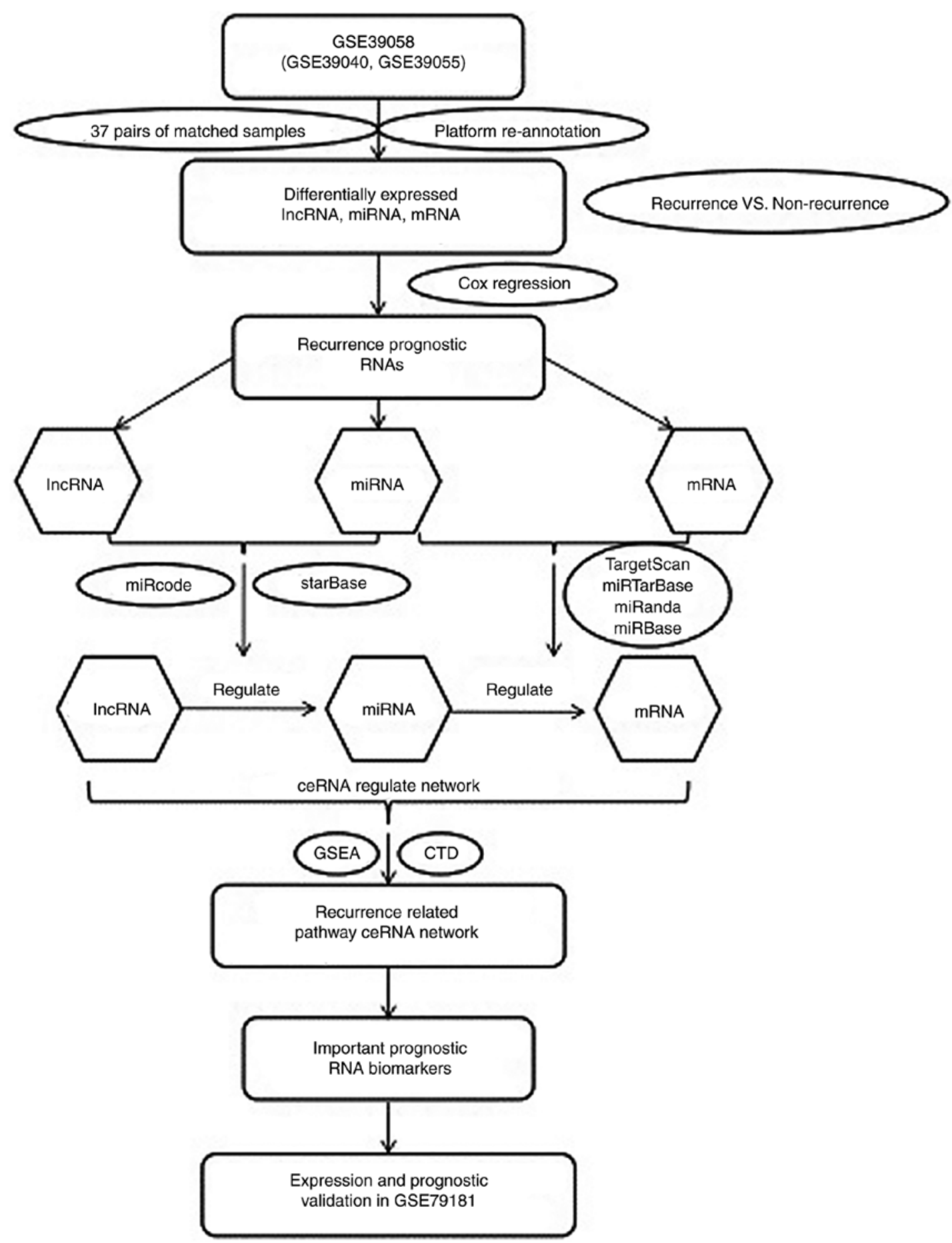

Figure 1. Flow chart of the analysis performed in the present study. GSE39058 was downloaded in order to identify prognosis-associated lncRNAs, miRNAs and mRNAs for ceRNA regulatory network construction. Important pathways were searched using GSEA. Kaplan-Meier survival analysis was conducted to determine the associations between the expression levels and survival prognosis. In addition, another independent dataset GSE79181 was also downloaded for validation. GSEA, Gene Set Enrichment Analysis; lncRNA, long non-coding RNA; miRNA, microRNA; ceRNA, competing endogenous RNA; CTD, Comparative Toxicogenomics Database.

pathways (34). Subsequently, the KEGG pathways directly associated with osteosarcoma were screened by searching the term 'osteosarcoma' in the Comparative Toxicogenomics Database (CTD; 2017 update; ctd.mdibl.org/) (35).

Validation of prognosis-associated RNAs. The important RNAs participating in the KEGG pathways directly associated with osteosarcoma were used to classify the samples into high- and low-expression groups based on the expression levels. Kaplan-Meier survival analysis, conducted with R software (version 3.4.1), was used to determine the associations between the expression levels and survival prognosis. Another independent miRNA profile, GSE79181, containing 25 osteosarcoma samples was downloaded from GEO (www.ncbi. nlm.nih.gov/geo/) to validate the reliability of the important molecules. 

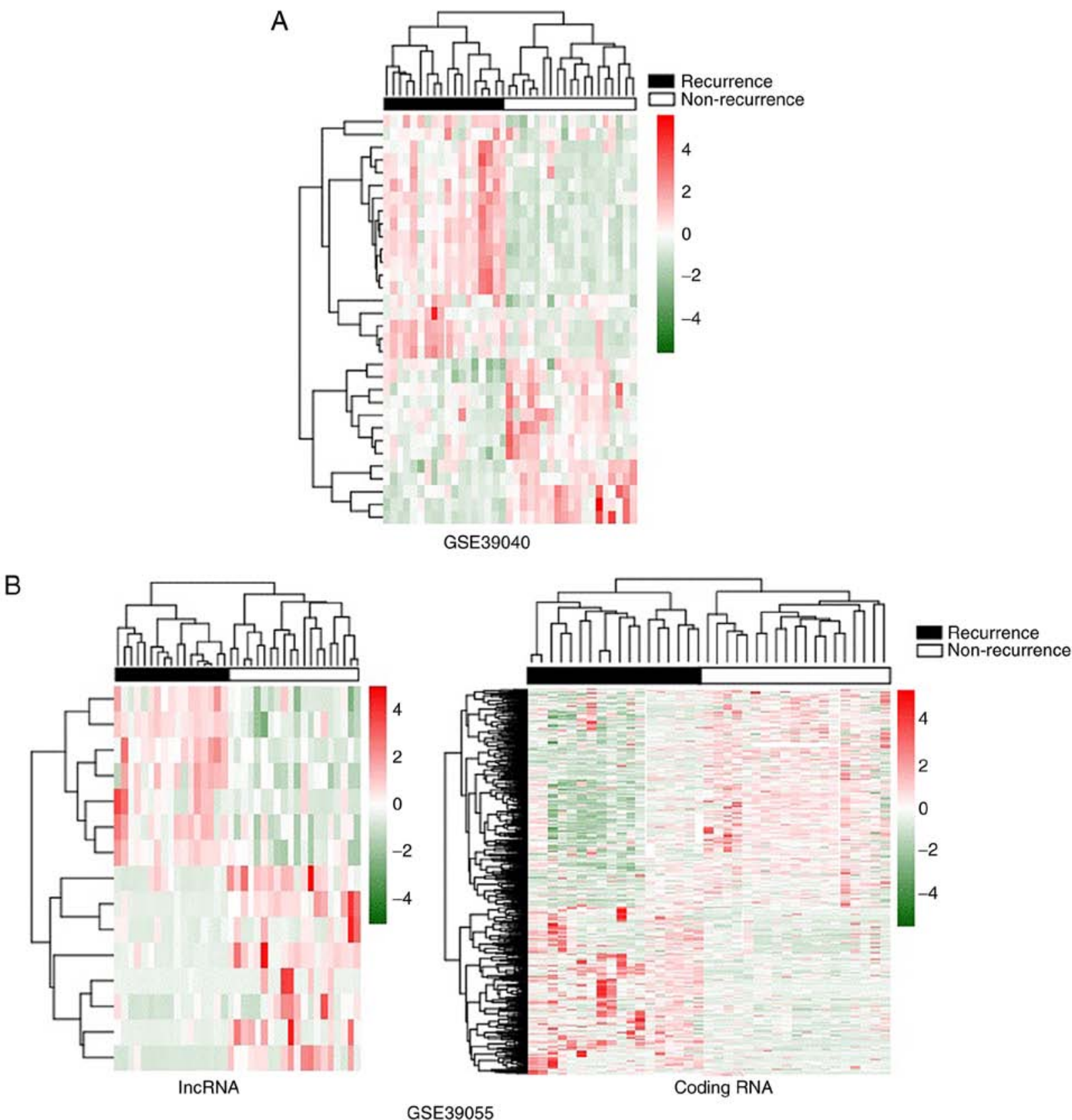

Figure 2. Hierarchical clustering analysis of screened differentially expressed RNAs. The color contrast indicates the marked differences in the expression levels between the recurrent and non-recurrent osteosarcoma samples. (A) Heatmap for GSE39040 using the differentially expressed miRNAs. (B) Heatmap for GSE39055 using the differentially expressed lncRNAs and mRNAs separately. IncRNA, long non-coding RNA; miRNA, microRNA.

\section{Results}

DEGs screening and hierarchical clustering. Following data preprocessing, 15 differentially expressed lncRNAs (8 downregulated and 7 upregulated lncRNAs) and 837 differentially expressed mRNAs (362 downregulated and 475 upregulated mRNAs) were identified in the GSE30955 profile. In addition, 32 differentially expressed miRNAs, including 13 downregulated and 19 upregulated miRNAs, were revealed in the GSE39040 profile. The expression values of differentially expressed miRNAs, IncRNAs, and mRNAs were hierarchically clustered, and the color contrast indicated a significant difference in the expression levels between the recurrent and non-recurrent osteosarcoma samples (Fig. 2).
Prognosis-associated mRNAs, miRNAs and lncRNAs. Among the selected differentially expressed RNAs, 417 RNAs (5 lncRNAs, 19 miRNAs and 393 mRNAs) were revealed to be associated with prognosis. The functional enrichment analysis for prognosis-associated mRNAs demonstrated that these 393 mRNAs were markedly involved with 10, 8 and $2 \mathrm{GO}$ terms in the $\mathrm{BP}, \mathrm{CC}$ and MF categories, respectively (Table I). These functions were mainly associated with 'chromatin organization' and 'transcription regulator'. Furthermore, 9 KEGG pathways were enriched for prognosis-associated mRNAs, such as 'transforming growth factor- $\beta$ signaling pathway' $(\mathrm{P}=0.0258)$, 'Wnt signaling pathway' $(\mathrm{P}=0.0366)$ and 'Oxidative phosphorylation' $(\mathrm{P}=0.0493$; Table II). 
Table I. Gene Ontology functional enrichment analysis in terms of biology process, cellular component and molecular function for the prognosis-associated mRNAs.

\begin{tabular}{|c|c|c|c|c|}
\hline Category & Term & Count & P-Value & Genes \\
\hline \multirow[t]{10}{*}{ BP } & $\begin{array}{l}\text { GO:0043009 chordate embryonic } \\
\text { development }\end{array}$ & 16 & 0.0033 & $\begin{array}{l}\text { MAFG, NBN, SYVN1, ADA, HOXA2, HOXA3, HOXC9, } \\
\text { MEOX2, HOXA5, HOXB8, NCOA6, APBA3, DAD1, } \\
\text { MAPK8IP3, ROR2, MED1 }\end{array}$ \\
\hline & $\begin{array}{l}\text { GO:0048568 embryonic organ } \\
\text { development }\end{array}$ & 10 & 0.0086 & $\begin{array}{l}\text { HOXA2, HOXC9, HOXA3, HOXB8, HOXA5, MYO7A, } \\
\text { ROR2, VAX2, ADA, MED1 }\end{array}$ \\
\hline & GO:0003002 regionalization & 10 & 0.0195 & $\begin{array}{l}\text { HOXA2, HOXC9, HOXA3, BTG2, MEOX2, HOXB8, } \\
\text { HOXA5, ROR2, VAX2, LRP5 }\end{array}$ \\
\hline & $\begin{array}{l}\text { GO:0051276 chromosome } \\
\text { organization }\end{array}$ & 18 & 0.0208 & $\begin{array}{l}\text { MEAF6, NBN, HIST1H1D, HIST1H2BF, HP1BP3, } \\
\text { HIST1H1A, EZH2, CENPE, TSPYL1, CHMP1A, EYA2, } \\
\text { HDAC2, NCAPG, PRDM5, CHD1,TLK1, RNF40, KDM5D }\end{array}$ \\
\hline & $\begin{array}{l}\text { GO:0001501 skeletal system } \\
\text { development }\end{array}$ & 13 & 0.0296 & $\begin{array}{l}\text { ESRRA, AEBP1, BMP1, TUFT1, ATP6V1B1, HOXA2, } \\
\text { HOXA3, HOXC9, HOXB8, HOXA5, ETS2, COMP, ROR2 }\end{array}$ \\
\hline & $\begin{array}{l}\text { GO:0006351 transcription, } \\
\text { DNA-dependent }\end{array}$ & 12 & 0.0367 & $\begin{array}{l}\text { TAF11, TAF1B, MED7, CCNK, TAF1A, ETS1, ANG, } \\
\text { NCOA6, NFIX, POLR2D, MED1, TAF1L }\end{array}$ \\
\hline & $\begin{array}{l}\text { GO:0032774 RNA biosynthetic } \\
\text { process }\end{array}$ & 12 & 0.0399 & $\begin{array}{l}\text { TAF11, TAF1B, MED7, CCNK, TAF1A, ETS1, ANG, } \\
\text { NCOA6, NFIX, POLR2D, MED1, TAF1L }\end{array}$ \\
\hline & $\begin{array}{l}\text { GO:0045597 positive regulation } \\
\text { of cell differentiation }\end{array}$ & 10 & 0.0448 & $\begin{array}{l}\text { IKZF1, ETS1, VHL, MAPT, KITLG, SEMA4D, SOCS5, } \\
\text { NTN1, BOC, ADA }\end{array}$ \\
\hline & $\begin{array}{l}\text { GO:0006325 chromatin } \\
\text { organization }\end{array}$ & 14 & 0.0458 & $\begin{array}{l}\text { MEAF6, HIST1H1D, HP1BP3, HIST1H2BF, HIST1H1A, } \\
\text { EZH2, TSPYL1, EYA2, HDAC2, PRDM5, CHD1, TLK1, } \\
\text { RNF40, KDM5D }\end{array}$ \\
\hline & $\begin{array}{l}\text { GO:0048598 embryonic } \\
\text { morphogenesis }\end{array}$ & 12 & 0.0495 & $\begin{array}{l}\text { HOXA2, EYA2, HOXC9, HOXA3, HOXB8, HOXA5, } \\
\text { MYO7A, CRABP2, ROR2, VAX2, MED1, LRP5 }\end{array}$ \\
\hline \multirow[t]{8}{*}{$\mathrm{CC}$} & GO:0044463 cell projection part & 15 & 0.0002 & $\begin{array}{l}\text { PLD2, BBS5, PARD3, TTLL6, KLC2, GAS8, ADA, } \\
\text { SPAG16, PCSK1, CC2D2A, CYBRD1, MAPK8IP3, } \\
\text { PEBP1, SLC5A6, CNTNAP1 }\end{array}$ \\
\hline & GO:0042995 cell projection & 26 & 0.0017 & $\begin{array}{l}\text { BBS5, PARD3, MYO7A, CLSTN1, TTLL6, KLC2, ADA, } \\
\text { SPAG16, PCSK1, ANG, MAPT, SLC4A7, CNTNAP1, } \\
\text { PLD2, DBNL, ARHGEF7, NPY1R, GAS8, THY1, GRM2, } \\
\text { CC2D2A, CYBRD1, PEBP1, MAPK8IP3, SLC5A6, GAP43 }\end{array}$ \\
\hline & GO:0005694 chromosome & 19 & 0.0030 & $\begin{array}{l}\text { TCP1, NBN, HIST1H1D, PRPF4B, IKZF1, HIST1H2BF, } \\
\text { PPP2R5A, HP1BP3, HIST1H1A, AKAP8L, CENPE, } \\
\text { TRIM66, CHMP1A, HDAC2, RIF1, NCAPG, PPP2CB, } \\
\text { CHD1, RNF40 }\end{array}$ \\
\hline & GO:0044427 chromosomal part & 15 & 0.0158 & $\begin{array}{l}\text { TCP1, NBN, HIST1H1D, IKZF1, HIST1H2BF, HP1BP3, } \\
\text { PPP2R5A, HIST1H1A, CENPE, TRIM66,HDAC2, RIF1, } \\
\text { NCAPG, PPP2CB, CHD1 }\end{array}$ \\
\hline & $\begin{array}{l}\text { GO:0005578 proteinaceous } \\
\text { extracellular matrix }\end{array}$ & 13 & 0.0195 & $\begin{array}{l}\text { ASPN, COL4A2, HMCN1, ADAMTSL2, LAMC3, ANG, } \\
\text { FBLN2, COMP, TIMP4, MMP3, NTN1, MFAP5, SPON1 }\end{array}$ \\
\hline & GO:0043005 neuron projection & 13 & 0.0306 & $\begin{array}{l}\text { ARHGEF7, NPY1R, KLC2, ADA, THY1, PCSK1, GRM2, } \\
\text { ANG, MAPT, MAPK8IP3, PEBP1, CNTNAP1, GAP43 }\end{array}$ \\
\hline & GO:0031012 extracellular matrix & 13 & 0.0322 & $\begin{array}{l}\text { ASPN, COL4A2, HMCN1, ADAMTSL2, LAMC3, ANG, } \\
\text { FBLN2, COMP, TIMP4, MMP3, NTN1, MFAP5, SPON1 }\end{array}$ \\
\hline & GO:0005730 nucleolus & 21 & 0.0440 & $\begin{array}{l}\text { UTP23, MEAF6, PNMA3, NBN, SYVN1, TAF1A, TSR1, } \\
\text { HNRNPA1, PNN, TSPYL1, NOP14, STAT6, DIS3, KRT17, } \\
\text { ANG, RPL9, NCOA6, PQBP1, LIAS, HNRNPH1, ARL4A }\end{array}$ \\
\hline MF & $\begin{array}{l}\text { GO:0043565 sequence-specific } \\
\text { DNA binding }\end{array}$ & 22 & 0.0116 & $\begin{array}{l}\text { MAFG, TSHZ3, ERF, ESRRA, IKZF1, TFE3, VAX2, NFIX, } \\
\text { ATF2, STAT6, ATF5, HOXA2, HDAC2, HOXA3, HOXC9, } \\
\text { MEOX2, HOXA5, ETS1,HOXB8, ETS2, PRDM5, CREB3L4 }\end{array}$ \\
\hline
\end{tabular}


Table I. Continued.

\begin{tabular}{|c|c|c|c|c|}
\hline Category & Term & Count & P-Value & Genes \\
\hline & $\begin{array}{l}\text { GO:0030528 transcription } \\
\text { regulator activity }\end{array}$ & 41 & 0.0440 & $\begin{array}{l}\text { TAF1B, TSHZ3, AEBP1, TAF1A, TFE3, E2F8, EZH2, } \\
\text { CNOT3, NFIX, MXI1, ATF2, ZNF75D, KCNIP3, STAT6, } \\
\text { TRIM66, HOXA2, HOXC9, HOXA3, HOXA5, PQBP1, } \\
\text { CREB3L4, TAF1L, MAFG, ERF, ESRRA, IKZF1, VAX2, } \\
\text { AFF1, MSRB2, TAF11, MED7, ATF5, HDAC2, MEOX2, } \\
\text { ETS1, HOXB8, ETS2, PRDM5, NCOA6, ID4, MED1 }\end{array}$ \\
\hline
\end{tabular}

GO, Gene Ontology; BP, biology process; CC, cellular component; MF, molecular function.

Table II. Nine enriched Kyoto Encyclopedia of Genes and Genomes pathways for prognosis-associated mRNAs.

\begin{tabular}{lccl}
\hline KEGG Pathway & Count & P-Value & \\
\hline $\begin{array}{l}\text { hsa00520:Amino sugar and nucleotide } \\
\text { sugar metabolism }\end{array}$ & 3 & 0.0224 & GMPPB, PGM3, FUK \\
hsa00603:Glycosphingolipid biosynthesis & 2 & 0.0249 & GBGT1, B3GALNT1 \\
hsa04350:TGF- $\beta$ signaling pathway & 4 & 0.0258 & AMHR2, COMP, PPP2CB, ID4 \\
hsa04144:Endocytosis & 6 & 0.0316 & PRKCZ, PLD2, PARD3, RAB11FIP3, ACAP2, AGAP1 \\
hsa04310:Wnt signaling pathway & 5 & 0.0366 & PPP2R5A, PPP2CB, PRICKLE2, PLCB1, LRP5 \\
hsa03020:RNA polymerase & 2 & 0.0437 & POLR2D, POLR2J2 \\
hsa04070:Phosphatidylinositol signaling system & 3 & 0.0445 & DGKQ, PLCD4, PLCB1 \\
hsa03040:Spliceosome & 4 & 0.0473 & PQBP1, MAGOHB, THOC2, HNRNPA1 \\
hsa00190:Oxidative phosphorylation & 4 & 0.0493 & NDUFB4, ATP6V1E2, ATP6V1B1, COX17 \\
\hline
\end{tabular}

KEGG, Kyoto Encyclopedia of Genes and Genomes; TGF- $\beta$, transforming growth factor- $\beta$.

ceRNA regulatory network construction and analysis. A total of 65 lncRNA-miRNA regulatory relationships associated with the 5 prognosis-associated lncRNAs were identified in miRcode and starBase. In addition, 7 lncRNA-miRNA regulatory relationships associated with the 19 prognosis-associated miRNAs were further screened, which involved three lncRNAs [long intergenic non-protein coding RNA 28 (LINC00028), LINC00323 and SNHG1] and four miRNAs (hsa-miR-124, hsa-miR-139-5p, hsa-miR-7, and hsa-miR-206). Furthermore, 172 miRNA-mRNA regulatory relationships were obtained for these four miRNAs using TargetScan, miRBase, miRanda, and miRTarBase. Finally, the 7 lncRNA-miRNA and 172 miRNA-mRNA regulatory relationships were used to construct the ceRNA regulatory network, which included 3 lncRNAs, 4 miRNAs and 143 mRNAs (Fig. 3).

The GSEA for the ceRNA regulatory network revealed that three KEGG pathways ['Mitogen-activated protein kinase (MAPK) signaling pathway', 'Chemokine signaling pathway' and 'Spliceosome'] were markedly associated with osteosarcoma (Table III). Furthermore, 'MAPK signaling pathway' involving three genes [Ras-related protein Rap-1b (RAP1B), activating transcription factor 2 (ATF2) and protein phosphatase $\mathrm{Mg}^{2+} / \mathrm{Mn}^{2+}$ dependent $1 \mathrm{~B}$ (PPM1B)] was also demonstrated to be associated with osteosarcoma by searching the CTD. Based on the ceRNA regulatory network, two
miRNAs (hsa-miR-124 and hsa-miR-7) and three lncRNAs (LINC00028, LINC00323, and SNHG1) could regulate the three mRNAs (RAP1B, ATF2, and PPM1B) that participate in the MAPK signaling pathway (Fig. 4).

Validation of prognosis-associated RNAs. The samples were classified into high- and low-expression groups based on the expression levels of three mRNAs (RAP1B, ATF2, and PPM1B), two miRNAs (hsa-miR-124 and hsa-miR-7), and three IncRNAs (LINC00028, LINC00323, and SNHG1). Kaplan-Meier survival analysis revealed that samples with lower expression levels of LINC00323 and SNHG1 had better prognosis, and increased expression levels of LINC00028, hsa-miR-124, and hsa-miR-7 were associated with better prognosis. The overexpression of RAP1B, ATF2 and PPM1B was also positively associated with osteosarcoma recurrence (Fig. 5).

The expression level of hsa-miR-7 was significantly downregulated in the osteosarcoma recurrent samples $(\mathrm{P}=0.02346)$ of another independent miRNA profile, GSE79181 (Fig. 6A). Kaplan-Meier survival analysis revealed that hsa-miR-124 $(\mathrm{P}=0.04681)$ and hsa-miR-7 $(\mathrm{P}=0.04853)$ were markedly associated with osteosarcoma recurrence (Fig. 6) using GSE79181. These results were in accordance with the GSE39040 analysis results. 
Table III. Gene Set Enrichment Analysis for the competing endogenous RNA regulatory network.

\begin{tabular}{lcccl}
\hline Name & $\begin{array}{c}\text { Enrichment } \\
\text { score }\end{array}$ & $\begin{array}{c}\text { Normalized } \\
\text { enrichment score }\end{array}$ & $\begin{array}{c}\text { Nominal } \\
\text { P-value }\end{array}$ & Genes \\
\hline MAPK_SIGNALING_PATHWAY & 0.5145 & 1.1238 & 0.0324 & RAP1B, ATF2, PPM1B \\
CHEMOKINE_SIGNALING_PATHWAY & 0.4710 & 1.0218 & 0.0423 & RAP1B, PLCB1, PRKCZ \\
SPLICEOSOME & 0.3188 & 0.6929 & 0.0485 & MAGOHB, PQBP1 \\
\hline
\end{tabular}

MAPK, mitogen-activated protein kinase; RAP1B, Ras-related protein Rap-1b; ATF2, activating transcription factor 2; PPM1B, protein phosphatase $\mathrm{Mg}^{2+} / \mathrm{Mn}^{2+}$ dependent 1B; PLCB1, phospholipase C $\beta 1$; PRKCZ, protein kinase C $\zeta$; MAGOHB, Mago homolog B exon junction complex subunit; PQBP1, polyglutamine binding protein 1 .

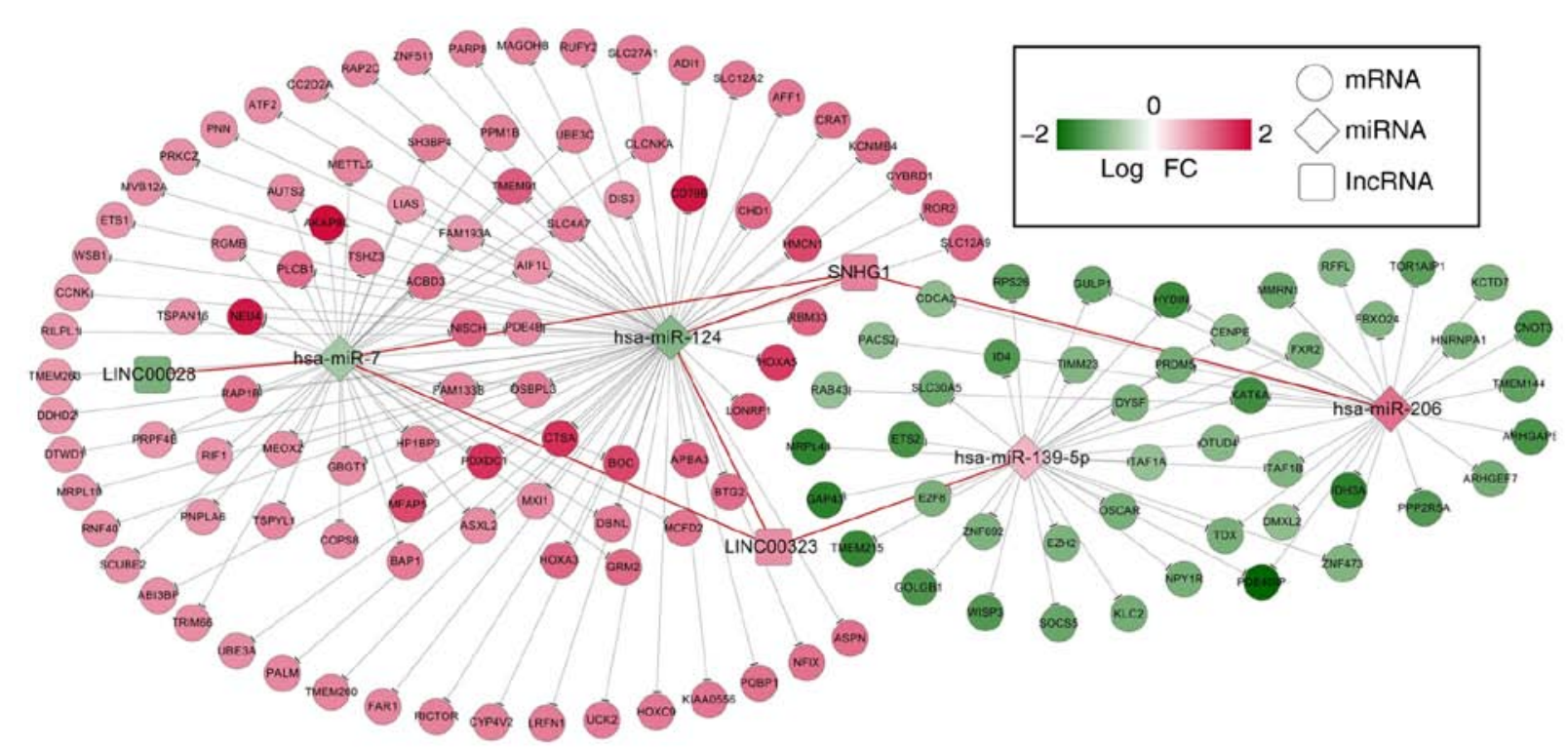

Figure 3. Constructed ceRNA regulatory network using prognosis-associated RNAs. The ceRNA regulatory network included 7 lncRNA-miRNA and 172 miRNA-mRNA regulatory associations, which involved 3 nncRNAs, 4 miRNAs and 143 mRNAs. Red nodes indicate the upregulated RNAs, and green indicate the downregulated RNAs. lncRNA, long non-coding RNA; miRNA/miR, microRNA; ceRNA, competing endogenous RNA; FC, fold change.

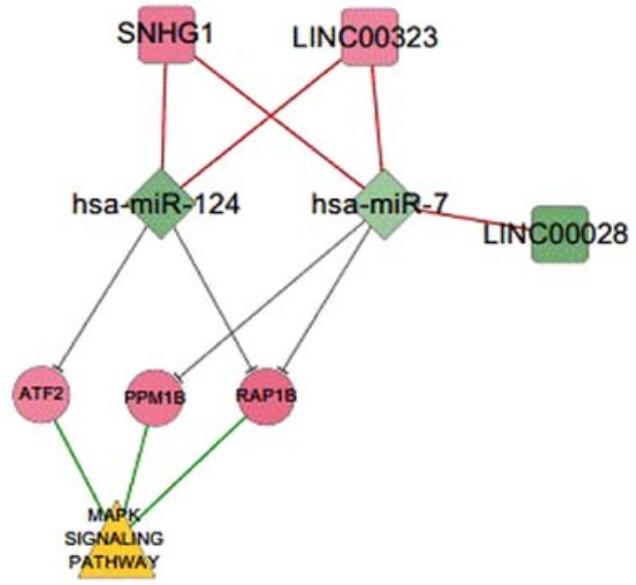

Figure 4. ceRNA regulatory network associated with the MAPK signaling pathway. Two miRNAs (hsa-miR-124 and hsa-miR-7) and three lncRNAs (LINC00028, LINC00323, and SNHG1) could regulate three mRNAs (RAP1B, ATF2 and PPM1B) that participate in the MAPK signaling pathway. IncRNA, long non-coding RNA; miRNA/miR, microRNA; ceRNA, competing endogenous RNA; LINC, long intergenic non-protein coding RNA; SNHG1, small nucleolar RNA host gene 1; RAP1B, Ras-related protein Rap-1b; ATF2, activating transcription factor 2; PPM1B, protein phosphatase $\mathrm{Mg}^{2+} / \mathrm{Mn}^{2+}$ dependent $1 \mathrm{~B} ; \mathrm{MAPK}$, mitogen-activated protein kinase.

\section{Discussion}

Understanding the mechanisms underlying osteosarcoma pathogenesis is necessary to determine novel biomarkers for the development of novel treatment strategies, particularly for metastases, as the prognosis of osteosarcoma is still poor (36). A number of previous studies have suggested that the dysregulated expression of IncRNAs could independently predict the outcomes of patients with osteosarcoma $(37,38)$. In the present study, 417 RNAs (5 lncRNAs, 19 miRNAs and 393 mRNAs) were revealed to be associated with prognosis among the total number of differentially expressed RNAs identified. Subsequently, the prognosis-associated RNAs were used to construct a ceRNA regulatory network for GSEA. The GSEA for the ceRNA regulatory network revealed that 'MAPK signaling pathway', 'Chemokine signaling pathway' and 'Spliceosome' were markedly associated with osteosarcoma. In addition, the MAPK signaling pathway was also demonstrated to be associated with osteosarcoma via the CTD.

MAPK is an insulin-mitogen activated protein (Ser/Thr) kinase, and activated MAPK can transmit extracellular signals to regulate cell growth, migration and apoptosis (39). Many 
A

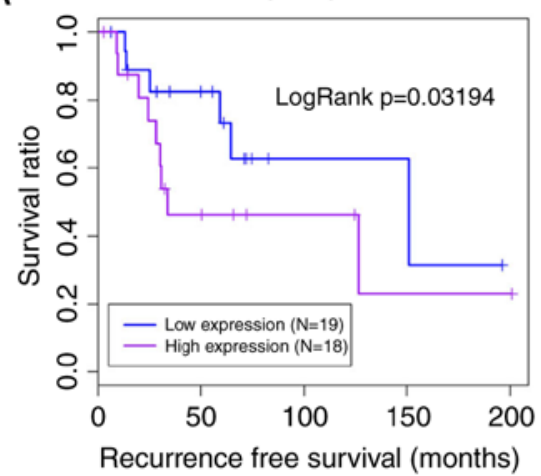

LINC00028

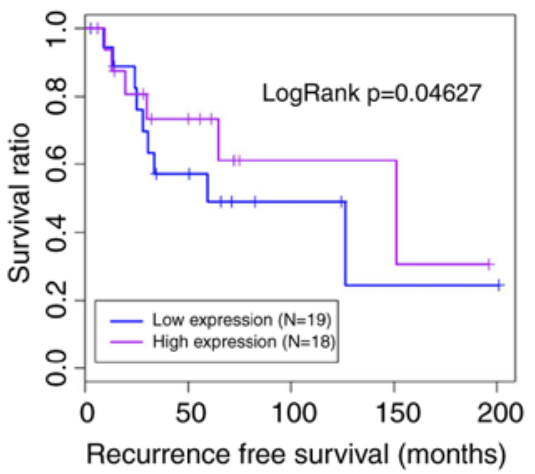

LINC00323

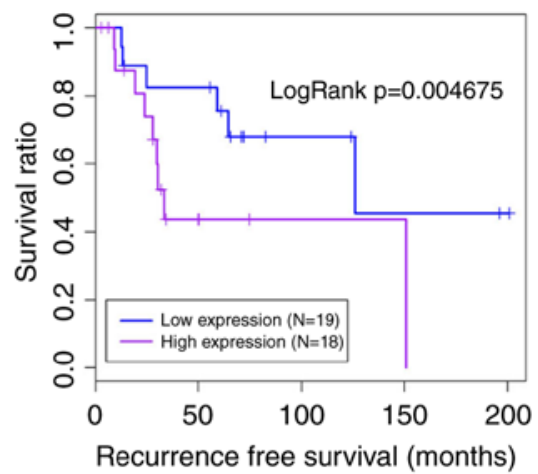

B

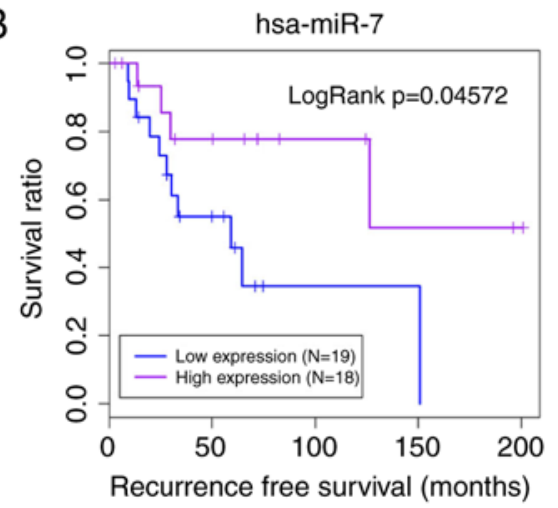

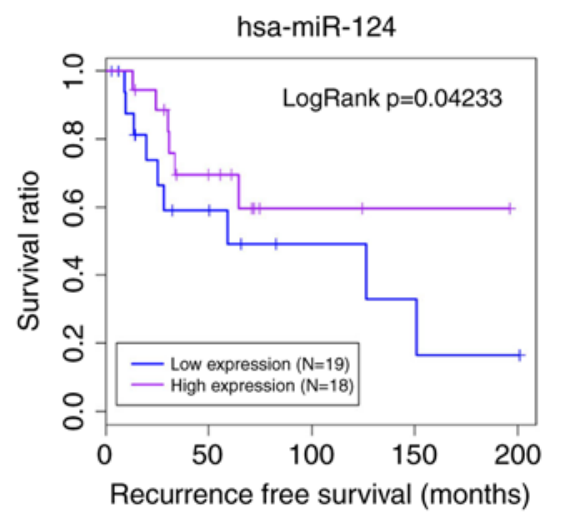
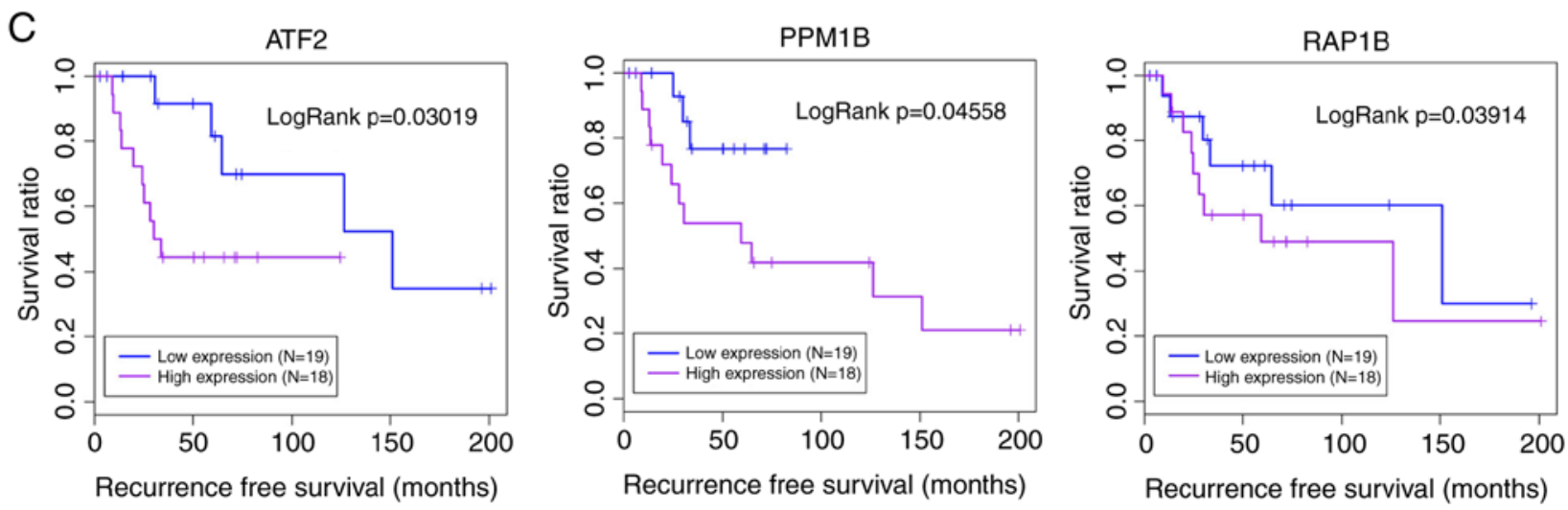

Figure 5. Kaplan-Meier survival analysis for lncRNAs, miRNAs and mRNAs associated with the mitogen-activated protein kinase signaling pathway. (A) Lower expression levels of LINC00323 and SNHG1, and increased expression levels of LINC00028 were associated with better prognosis. (B) Samples with increased expression levels of hsa-miR-124 and hsa-miR-7 had better prognosis. (C) The overexpression of RAP1B, ATF2, and PPM1B was positively associated with osteosarcoma recurrence. IncRNA, long non-coding RNA; miRNA/miR, microRNA; ceRNA, competing endogenous RNA; LINC, long intergenic non-protein coding RNA; SNHG1, small nucleolar RNA host gene 1; RAP1B, Ras-related protein Rap-lb; ATF2, activating transcription factor 2; PPM1B, protein phosphatase $\mathrm{Mg}^{2+} / \mathrm{Mn}^{2+}$ dependent 1B.

studies have reported the significant roles of the MAPK signaling pathway in osteosarcoma $(40,41)$. The expression level of S100 calcium binding protein A9 (S100A9) was increased in human osteosarcoma tissues and associated with survival rate, and the downregulation of S100A9 inhibited osteosarcoma cell proliferation through the inactivation of the MAPK and NF- $\kappa B$ signaling pathways (42). Increased expression levels of phospholipase A2 group XVI could enhance osteosarcoma metastasis by activating the MAPK signaling pathway (43). Delphinidin treatment could inhibit cell migration and prevented epithelial-to-mesenchymal transition via the MAPK signaling pathway in osteosarcoma cell lines (44). Onzin overexpression could also contribute to more aggressive osteosarcoma metastatic phenotypes through the C-X-C motif chemokine 5-MAPK signaling pathway (45).

In the constructed ceRNA regulatory network, three IncRNAs (LINC00028, LINC00323 and SNHG1) and two miRNAs (hsa-miR-124 and hsa-miR-7) regulating three mRNAs (RAP1B, ATF2 and PPM1B) participated in the MAPK signaling pathway. In addition, samples with increased expression levels of hsa-miR-124 and hsa-miR-7 had better prognosis. Expression levels of miR-124 in the metastatic osteosarcoma tissues were revealed to be significantly lower than those observed in non-metastatic tissues, and miR-124 could function as a tumor suppressor miRNA in osteosarcoma through the inhibition of Rac 
A

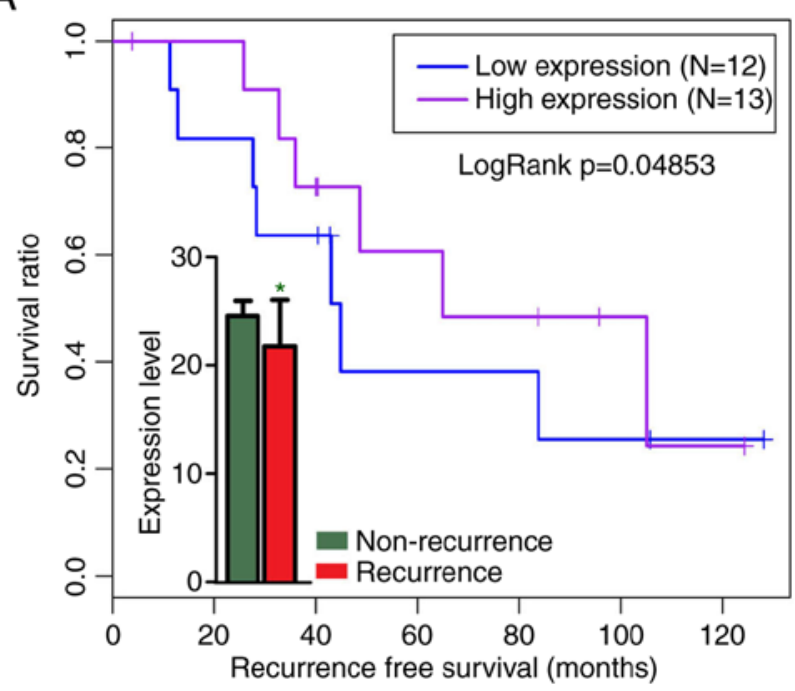

B

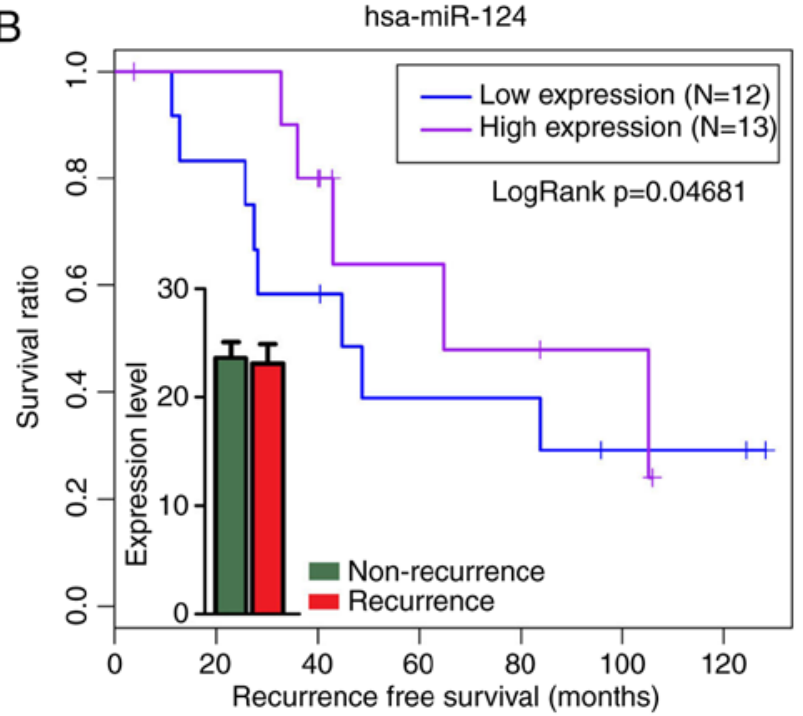

Figure 6. Kaplan-Meier survival analysis of hsa-miR-124 and hsa-miR-7 with osteosarcoma recurrence using GSE79181 for validation. (A) hsa-miR-7 was significantly downregulated in the osteosarcoma recurrent samples. (B) hsa-miR-124 was markedly associated with osteosarcoma recurrence. Data are presented as the mean \pm standard deviation. ${ }^{*} \mathrm{P}<0.05$ vs. non-recurrence. miR, microRNA.

family small guanosine triphosphatase 1 expression (46). In addition, miR-7 expression was decreased in osteosarcoma tissues and low miR-7 expression was associated with poor prognosis (47). Furthermore, the roles of hsa-miR-124 and hsa-miR-7 in osteosarcoma recurrence were also validated using another independent miRNA profile in the present study.

Kaplan-Meier survival analysis revealed that samples with lower expression levels of LINC00323 and SNHG1 had better prognosis, which were consistent with a study by Wang et al (48), which demonstrated that SNHG1 was upregulated in osteosarcoma tissues and cell lines, and high SNHG1 expression predicted poor overall survival in patients with osteosarcoma; these findings were consistent with the present results. Wang et al (48) reported that SNHG1 increased human nin one binding protein (an oncogene) by acting as a ceRNA to sponge miR-326, promoting cell growth, migration and invasion in osteosarcoma, while the present study revealed that SNHG1 increased ATF2, PPM1B and RAP1B by sponging miR-124 and miR-7 as a ceRNA. In addition, high SNHG1 expression was also positively associated with the metastasis of osteosarcoma, and miR-577 could act as a ceRNA of SNHG1 by targeting WNT2B which served an oncogenic role in osteosarcoma cells by activating the Wnt/ $\beta$-catenin signaling pathway (49). Therefore, the present study showed that the SNHG1/miR-124 and miR-7/RAP1B, ATF2 and PPM1B/MAPK signaling pathway regulatory network may provide a potential novel therapeutic strategy for osteosarcoma treatment.

In conclusion, three IncRNAs (LINC00028, LINC00323 and SNHG1) and two miRNAs (hsa-miR-124 and hsa-miR-7) regulating three mRNAs (RAP1B, ATF2 and PPM1B) participated in MAPK signaling pathway, as identified by the ceRNA regulatory network, and were associated with osteosarcoma recurrence. However, the prognosis prediction value based on the expressions of these important RNAs still requires further confirmation based on clinical experiments in an independent cohort of patients with non-recurrent and recurrent osteosarcoma.

\section{Acknowledgements}

Not applicable.

\section{Funding}

No funding was received.

\section{Availability of data and materials}

The datasets used and/or analyzed during the present study are available from the corresponding author on reasonable request.

\section{Authors' contributions}

SZ and HF contributed to the study design. SZ, LD, XL and HF searched for and downloaded the gene expression profiles from the Gene Expression Omnibus database. SZ, LD, XL and HF performed the analysis and interpretation of the microarray dataset. SZ and HF critically revised the manuscript for important intellectual content. All authors read and approved the final manuscript.

\section{Ethics approval and consent to participate}

Not applicable.

\section{Patient consent for publication}

Not applicable.

\section{Competing interests}

The authors declare that they have no competing interests. 


\section{References}

1. Geller DS and Gorlick R: Osteosarcoma: A review of diagnosis, management, and treatment strategies. Clin Adv Hematol Oncol 8: 705-718, 2010

2. He H, Ni J and Huang J: Molecular mechanisms of chemoresistance in osteosarcoma (Review). Oncol Lett 7: 1352-1362, 2014.

3. Liu JJ, Liu S, Wang JG, Zhu W, Hua YQ, Sun W and Cai ZD: Telangiectatic osteosarcoma: A review of literature. Onco Targets Ther 6: 593-602, 2013.

4. McQueen P, Ghaffar S, Guo Y, Rubin EM, Zi X and Hoang BH: The Wnt signaling pathway: Implications for therapy in osteosarcoma. Expert Rev Anticancer Ther 11: 1223-1232, 2011.

5. Lin $\mathrm{CH}$, Ji T, Chen CF and Hoang BH: Wnt signaling in osteosarcoma. Adv Exp Med Biol 804: 33-45, 2014.

6. McManus MM, Weiss KR and Hughes DP: Understanding the role of Notch in osteosarcoma. Adv Exp Med Biol 804: 67-92, 2014.

7. Graziano AC, Cardile V, Avola R, Vicario N, Parenti C, Salvatorelli L, Magro G and Parenti R: Wilms' tumor gene 1 silencing inhibits proliferation of human osteosarcoma MG-63 cell line by cell cycle arrest and apoptosis activation. Oncotarget 8: 13917-13931, 2017.

8. Zhang F, Yan T, Guo W, Sun K, Wang S, Bao X, Liu K, Zheng B, Zhang $\mathrm{H}$ and Ren T: Novel oncogene COPS3 interacts with beclin1 and Raf-1 to regulate metastasis of osteosarcoma through autophagy. J Exp Clin Cancer Res 37: 135, 2018

9. Chan LH, Wang W, Yeung W, Deng Y, Yuan P and Mak KK: Hedgehog signaling induces osteosarcoma development through Yap1 and H19 overexpression. Oncogene 33: 4857-4866, 2014.

10. Zhou S, Yu L, Xiong M and Dai G: LncRNA SNHG12 promotes tumorigenesis and metastasis in osteosarcoma by upregulating Notch2 by sponging miR-195-5p. Biochem Biophys Res Commun 495: 1822-1832, 2018.

11. Wang Y, Zhao Z, Zhang S, Li Z, Li D, Yang S, Zhang H, Zeng X and Liu J: LncRNA FAL1 is a negative prognostic biomarker and exhibits pro-oncogenic function in osteosarcoma. J Cell Biochem 119: 8481-8489, 2018.

12. Tian ZZ, Guo XJ, Zhao YM and Fang Y: Decreased expression of long non-coding RNA MEG3 acts as a potential predictor biomarker in progression and poor prognosis of osteosarcoma. Int J Clin Exp Pathol 8: 15138-15142, 2015.

13. Wang Y and Kong D: Knockdown of lncRNA MEG3 inhibits viability, migration, and invasion and promotes apoptosis by sponging miR-127 in osteosarcoma cell. J Cell Biochem 119: 669-679, 2018

14. Zhou Q, Chen F, Fei Z, Zhao J, Liang Y, Pan W, Liu X and Zheng D: Genetic variants of lncRNA HOTAIR contribute to the risk of osteosarcoma. Oncotarget 7: 19928-19934, 2016.

15. Ma B, Li M, Zhang L, Huang M, Lei JB, Fu GH, Liu CX, Lai QW, Chen QQ and Wang YL: Upregulation of long non-coding RNA TUG1 correlates with poor prognosis and disease status in osteosarcoma. Tumour Biol 37: 4445-4455, 2016.

16. Li F, Cao L, Hang D, Wang F and Wang Q: Long non-coding RNA HOTTIP is up-regulated and associated with poor prognosis in patients with osteosarcoma. Int J Clin Exp Pathol 8: 11414-11420, 2015

17. Salmena L, Poliseno L, Tay Y, Kats L and Pandolfi PP: A ceRNA hypothesis: The rosetta stone of a hidden RNA language? Cell 146: 353-358, 2011

18. Kelly AD, Haibe-Kains B, Janeway KA, Hill KE, Howe E, Goldsmith J, Kurek K, Perez-Atayde AR, Francoeur N, Fan JB, et al: MicroRNA paraffin-based studies in osteosarcoma reveal reproducible independent prognostic profiles at $14 \mathrm{q} 32$. Genome Med 5: 2, 2013.

19. Bolstad B: PreprocessCore: A collection of pre-processing functions. R package version 1, 2013.

20. Smyth GK: Limma: Linear models for microarray data. Bioinformatics computational biology Solutions Using $\mathrm{R}$ Bioconductor, Springer 397-420, 2005.

21. Rao Y, Lee Y, Jarjoura D, Ruppert AS, Liu CG, Hsu JC and Hagan JP: A comparison of normalization techniques for microRNA microarray data. Stat Appl Genet Mol Biol 7: Article22, 2008

22. Yates B, Braschi B, Gray KA, Seal RL, Tweedie S and Bruford EA: Genenames.org: The HGNC and VGNC resources in 2017. Nucleic Acids Res 45: D619-D625, 2017.
23. Benjamini Y and Hochberg Y: Controlling the false discovery rate: A practical and powerful approach to multiple testing. J Royal Stat Soc Series B 57: 289-300, 1995.

24. Wang L, Cao C, Ma Q, Zeng Q, Wang H, Cheng Z, Zhu G, Qi J, Ma H, Nian H and Wang Y: RNA-seq analyses of multiple meristems of soybean: Novel and alternative transcripts, evolutionary and functional implications. BMC Plant Biol 14: 169, 2014.

25. Gosling C: Encyclopedia of distances. Reference Rev 24: 1-583, 2009.

26. Wang $\mathrm{P}$, Wang $\mathrm{Y}$, Hang $\mathrm{B}$, Zou $\mathrm{X}$ and Mao JH: A novel gene expression-based prognostic scoring system to predict survival in gastric cancer. Oncotarget 7: 55343-55351, 2016.

27. Huang Da W, Sherman BT and Lempicki RA: Systematic and integrative analysis of large gene lists using DAVID bioinformatics resources. Nature Protoc 4: 44-57, 2009.

28. Li JH, Liu S, Zhou H, Qu LH and Yang JH: starBase v2.0: Decoding miRNA-ceRNA, miRNA-ncRNA and protein-RNA interaction networks from large-scale CLIP-Seq data. Nucleic Acids Res 42: D92-D97, 2014.

29. Agarwal V, Bell GW, Nam JW and Bartel DP: Predicting effective microRNA target sites in mammalian mRNAs. Elife 12: 4, 2015.

30. Lee YY, Kim TJ, Kim JY, Choi CH, Do IG, Song SY, Sohn I, Jung SH, Bae DS, Lee JW and Kim BG: Genetic profiling to predict recurrence of early cervical cancer. Gynecol Oncol 131: 650-654, 2013

31. Jeggari A, Marks DS and Larsson E: miRcode: A map of putative microRNA target sites in the long non-coding transcriptome. Bioinformatics 28: 2062-2063, 2012.

32. Kozomara A and Griffiths-Jones S: miRBase: Annotating high confidence microRNAs using deep sequencing data. Nucleic Acids Res 42: D68-D73, 2014.

33. Shannon P, Markiel A, Ozier O, Baliga NS, Wang JT, Ramage D, Amin N, Schwikowski B and Ideker T: Cytoscape: A software environment for integrated models of biomolecular interaction networks. Genome Res 13: 2498-2504, 2003.

34. Subramanian A, Tamayo P, Mootha VK, Mukherjee S, Ebert BL, Gillette MA, Paulovich A, Pomeroy SL, Golub TR, Lander ES and Mesirov JP: Gene set enrichment analysis: A knowledge-based approach for interpreting genome-wide expression profiles. Proc Natl Acad Sci USA 102: 15545-15550, 2005.

35. Davis AP, Grondin CJ, Johnson RJ, Sciaky D, King BL, McMorran R, Wiegers J, Wiegers TC and Mattingly CJ: The comparative toxicogenomics database: Update 2017. Nucleic Acids Res 45: D972-D978, 2017.

36. He JP, Hao Y, Wang XL, Yang XJ, Shao JF, Guo FJ and Feng JX: Review of the molecular pathogenesis of osteosarcoma. Asian Pac J Cancer Prev 15: 5967-5976, 2014.

37. Wang B, Su Y, Yang Q, Lv D, Zhang W, Tang K, Wang H, Zhang R and Liu Y: Overexpression of long non-coding RNA HOTAIR promotes tumor growth and metastasis in human osteosarcoma. Mol Cells 38: 432-440, 2015.

38. Zhang Q, Geng PL, Yin P, Wang XL, Jia JP and Yao J: Down-regulation of long non-coding RNA TUG1 inhibits osteosarcoma cell proliferation and promotes apoptosis. Asian Pac J Cancer Prev 14: 2311-2325, 2013.

39. Sui X, Kong N, Ye L, Han W, Zhou J, Zhang Q, He C and Pan H: p38 and JNK MAPK pathways control the balance of apoptosis and autophagy in response to chemotherapeutic agents. Cancer Lett 344: 174-179, 2014.

40. Chen HJ, Lin CM, Lee CY, Shih NC, Peng SF, Tsuzuki M, Amagaya S, Huang WW and Yang JS: Kaempferol suppresses cell metastasis via inhibition of the ERK-p38-JNK and AP-1 signaling pathways in U-2 OS human osteosarcoma cells. Oncol Rep 30: 925-932, 2013.

41. Cheng DD, Zhu B, Li SJ, Yuan T, Yang QC and Fan CY: Down-regulation of RPS9 inhibits osteosarcoma cell growth through inactivation of MAPK signaling pathway. J Cancer 8: 2720-2728, 2017.

42. Cheng S, Zhang X, Huang N, Qiu Q, Jin Y and Jiang D: Down-regulation of S100A9 inhibits osteosarcoma cell growth through inactivating MAPK and NF- $\kappa \mathrm{B}$ signaling pathways. BMC Cancer 16: 253, 2016.

43. Li L, Liang S, Wasylishen AR, Zhang Y, Yang X, Zhou B, Shan L, Han X, Mu T, Wang G and Xiong S: PLA2G16 promotes osteosarcoma metastasis and drug resistance via the MAPK pathway. Oncotarget 7: 18021-18035, 2016. 
44. Kang HM, Park BS, Kang HK, Park HR, Yu SB and Kim IR Delphinidin induces apoptosis and inhibits epithelial-to-mesenchymal transition via the ERK/p38 MAPK-signaling pathway in human osteosarcoma cell lines. Environ Toxicol 33: 640-649, 2018.

45. Zhang Y, Hu Q, Li G, Li L, Liang S, Zhang Y, Liu J, Fan Z, Li L, Zhou B, et al: ONZIN upregulation by mutant p53 contributes to osteosarcoma metastasis through the CXCL5-MAPK signaling pathway. Cell Physiol Biochem 48: 1099-1111, 2018.

46. Geng S, Zhang X, Chen J, Liu X, Zhang H, Xu X, Ma Y, Li B, Zhang Y, Bi Z and Yang C: The tumor suppressor role of miR-124 in osteosarcoma. PLoS one 9: e91566, 2014.

47. Shouying L, Changxi Z, Changbao Z, Qiuhe S, Ming W, Ye L and Huaijie A: Low-expression of miR-7 promotes cell proliferation and exhibits prognostic value in osteosarcoma patients. Int J Clin Exp Pathol 10: 9035-9041, 2017.
48. Wang J, Cao L, Wu J and Wang Q: Long non-coding RNA SNHG1 regulates NOB1 expression by sponging miR-326 and promotes tumorigenesis in osteosarcoma. Int J Oncol 52: 77-88, 2018.

49. Jiang Z, Jiang $C$ and Fang J: Up-regulated lnc-SNHG1 contributes to osteosarcoma progression through sequestration of miR-577 and activation of WNT2B/Wnt/ $\beta$-catenin pathway. Biochem Biophys Res Commun 495: 238-245, 2018.

(i) () $\odot$ This work is licensed under a Creative Commons Attribution-NonCommercial-NoDerivatives 4.0 International (CC BY-NC-ND 4.0) License. 